INNOVACIÓN

\title{
El aprendizaje basado en investigación como alternativa didáctica del proceso de aprendizaje- enseñanza en el derecho: Una experiencia extracurricular en desarrollo
}

\author{
A aprendizagem baseada em pesquisa como alternativa didática ao processo \\ de ensino-aprendizagem no direito: Uma experiência extracurricular \\ em desenvolvimento
}

Learning based on research as didactic alternative of the teaching-learning process in law school: An extracurricular experience in development

\author{
Mauricio Andrés Figueroa Mendoza \\ Universidad Santo Tomas, Chile
}

\begin{abstract}
RESUMEN El presente trabajo se desarrolla a partir de tres aspectos. En primer lugar, se describe una problemática que influye directamente en el desarrollo y progresión de las competencias requeridas en estudiantes de Derecho y que resulta ser una debilidad para alcanzar el perfil de egreso. En la segunda parte se explora la opción didáctica del aprendizaje basado en investigación como una alternativa frente al desarrollo de habilidades en estudiantes de Derecho y se exploran las ventajas que éste presenta para alcanzar las competencias requeridas como abogados, a partir de la sistematización de la información seleccionada. Por último, se presentan algunas observaciones extraídas a partir de la experiencia, y se define la implementación de esta metodología fuera de un contexto tradicional del aula, a través de talleres extracurriculares impartidos en la Escuela de Derecho de la Universidad Santo Tomás en Antofagasta, lo que da cuenta de que el aprendizaje basado en investigación como estrategia metodológica puede ser aplicado en contextos extracurriculares con interesantes avances que se evidencian en el tiempo.
\end{abstract}

PALABRAS CLAVE Aprendizaje basado en investigación, investigación jurídica, proceso aprendizaje-enseñanza, perfil de egreso.

RESUMO O presente trabalho é desenvolvido com base em três aspectos. Em primeiro lugar, é feita uma descrição de um problema que influencia diretamente o desenvolvi- 
mento e a progressão das competências exigidas pelos estudantes de direito e que acaba por ser uma deficiência no perfil do estudante de graduação. Na segunda parte, a opção didática do aprendizagem baseada em pesquisa é explorada como uma alternativa ao desenvolvimento de habilidades em estudantes de direito e as vantagens que apresenta para alcançar as competências necessárias à medida que os advogados são explorados, principalmente a partir da sistematização das informações selecionadas. Finalmente, algumas observações extraídas da experiência são descritas e apresentadas, e a implementação dessa metodologia é definida fora do contexto tradicional da sala de aula, por meio de cursos extracurriculares ministradas na Faculdade de Direito da Universidade de Santo Tomás Antofagasta, perceber que o Aprendizagem baseada em pesquisa como estratégia metodológica pode ser aplicado em contextos extracurriculares com avanços interessantes que são evidentes ao longo do tempo.

PALAVRAS-CHAVE Aprendizagem baseada em pesquisa, pesquisa jurídica, processo ensino-aprendizagem, perfil de graduação.

ABSTRACT The present work is based on three aspects. Firstly, there was a description of a problem that directly affects the development and progression of competencies required of law students, which turns out to be a weakness in achieving the graduation profile. Secondly, there was an exploration of the didactic option of research-based learning as an alternative to developing skills in law students. Also, it explored the advantages of research-based learning to meet the required competencies of law education. A systematization of the selected information was conducted. Finally, there were a description and presentation of observations extracted from experience. The implementation of this methodology outside of a traditional classroom context involved extracurricular workshops held at the Law School of the Santo Tomás Antofagasta University. Findings suggested that research-based learning as a methodological strategy can be applied in extracurricular contexts with interesting advances that become evident throughout time.

KEYWORDS Research-based learning, legal research, teaching-learning process, graduation profile.

\section{Introducción y antecedentes teóricos}

Desde la década de 1980, las universidades chilenas han experimentado una serie de cambios (Cruz-Coke, 2004) debido al contexto social, político y económico de la época, lo que ha generado dos fenómenos que hasta hoy están presentes.

Por un lado, la proliferación de instituciones de educación superior, como los centros de formación técnica y los institutos profesionales - a lo que habría que agregar bajo el mismo contexto a las universidades privadas-, lo que significó la ampliación de la oferta en educación superior y sobre todo el evidente aumento de planteles uni- 
versitarios. De las 8 universidades existentes en el año 1980, se llegó a 31 en 1989, y a 70 universidades en 1999, número que siguió incrementando en el tiempo.

El segundo fenómeno tiene relación directa con el antedicho, esto es, la masificación de estudiantes que ingresan a universidades. De esta situación no escapa el derecho, ya que al alto número de ingresos a estas carreras del país, se suma el alto número de titulados (Lazo, 2011: 250), el que además ha aumentado en forma significativa en los últimos diez años. A esto se suman los efectos que eso conlleva, esto es, multiplicidad de realidades socioculturales, estudiantes provenientes de contextos educativos diversos, con conocimientos previos, habilidades y competencias de entrada totalmente heterogéneas (Coloma Correa y Agüero, 2012: 43), lo que se manifiesta en una población de estudiantes que ingresan con distintas características, pero que deben salir al campo laboral con competencias homogéneas que den cuenta del perfil de egreso como producto final (Benfeld, 2016: 145).

En el campo de Derecho, la coyuntura vivida desde los años ochenta a la fecha ha obligado a un cambio constante de planes y programas de las distintas escuelas (Benfeld, 2016: 145) que tratan de adaptar su oferta, su estructura de asignaturas y los paradigmas educativos a las necesidades que el mercado requiere. Esto se traduce hoy en que el norte de la escuela de Derecho está orientado hacia el perfil de egreso, es decir, lo que los estamentos han establecido finalmente como requerimiento del futuro abogado. Es en este contexto que se producen las más importantes desavenencias y desencuentros en las facultades y escuelas, entre lo que el mercado laboral requiere, lo que la escuela o facultad declara a través de su perfil de egreso y lo que al final pasa en el aula, en la práctica docente, en el proceso mismo de aprendizaje y enseñanza, el que cada docente ejecuta a su discreción, sin importar en ocasiones las habilidades a desarrollar o el perfil de egreso al cual tributar.

En este sentido, el gran obstáculo sigue siendo por una parte la clase tradicional, el carácter enciclopedista de la enseñanza jurídica (Solari, 2012: 704) y la clase magistral que tiene como única fuente de información (Coloma Correa, 2006: 125) la entregada por el docente, el que la presenta, por lo demás, como realidad inalterable en las aulas de Derecho (Böhmer, 2009: 233). Esta cuestión no es privativa de nuestro país, pues se reproduce en el resto de América Latina (Böhmer, 2009: 16).

La enseñanza tradicional del derecho da cuenta de un conductismo típico en la clase, según el cual el conocimiento entregado se basa en la enseñanza de normas por un lado y de conceptos por el otro, para desarrollar con ello habilidades mecánicas de respuesta, en que la memoria (Elgueta y Palma, 2015: 34) es el principal insumo del estudiante, medio que le permitirá alcanzar una mejor calificación en las evaluaciones a las que es sometido, aunque solo ha explorado habilidades de primer nivel, como el conocimiento (Anderson y Krathworhl, 2001), mientras deja muchas veces sin desarrollar habilidades de orden superior que no son trabajadas y mucho menos evaluadas. Lo anterior podría declararse como coherencia perfecta entre la 
clase y la evaluación, en las que se enseña conocimiento y se pregunta conocimiento, lo que implica un total desapego con el perfil de egreso y las competencias que debiera alcanzar el estudiante. En gran medida, estos resultados se explican por la forma tradicional y conductista, que se resiste a abandonar la práctica pedagógica en muchas de las salas de Derecho, además del modelo de enseñanza (Coloma Correa, 2005: 147) y su evaluación, la que finalmente podría significar la posibilidad de moldear a un estudiante pasivo, carente de ingenio para resolver situaciones distintas que aquéllas para las que ha sido mecanizado (De Bianchetti, Fridman y Zarate, 2003: 3). La estandarización del aprendizaje limita la creatividad y el ingenio para resolver problemas y aplicar en este caso las normas en situaciones concretas, lo que Joy Paul Guilford denominó como pensamiento convergente, el que necesariamente apunta a respuestas correctas y estandarizadas; ello en contraposición al pensamiento divergente que, como señala Camilloni (2019: 13), «se caracteriza por la fluidez (cantidad de respuestas), la flexibilidad (variedad de respuestas), la originalidad (novedad de respuestas), la sensibilidad para hallar e identificar problemas y la capacidad de elaborar y de transformar».

De lo anterior, no queda más que compartir lo que Benfeld (2016: 164) concluye cuando explica:

Los autores nacionales que han estudiado y escrito sobre la enseñanza del derecho, sin perjuicio de haber realizado notables aportaciones y avances en la discusión sobre los temas clásicos sobre enseñanza y aprendizaje, han dicho muy poco sobre la forma que el nuevo paradigma de universitario (masivo y comprometido con el mercado) influye en la enseñanza del derecho.

Centra así la mirada en el contexto exclusivo de lo que el mercado laboral requiere y en la tradición clásica de la enseñanza del derecho, que explora de manera aislada cuestiones metodológicas, curriculares y evaluativas, sin un contexto sistémico, modernizador y práctico que permita adecuar no solo la práctica de la enseñanza del derecho, sino que los planes y programas bajo un paradigma en que el estudiante y el abogado sean uno solo y no dos entidades disociadas en una misma persona. Como señala Molina (2009: 9): «No coinciden las expectativas sociales y políticas actuales con la parsimonia del operador jurídico que se contagia a la academia y el salón de clases».

Nos planteamos algunas interrogantes frente al escenario descrito, que incumbe por un lado la resistencia a abandonar por completo las prácticas pedagógicas implementadas en la enseñanza del derecho, y por otro la necesidad de coordinar estas prácticas o en su medida modificarlas para concretar el aprendizaje del estudiante en torno al perfil de egreso. Entonces, nos planteamos: ¿es posible soslayar la deficiencia que puede existir en la práctica pedagógica o en el proceso mismo de la educación del derecho respecto del desarrollo de habilidades que tributen al perfil de egreso? 
Para ello, una primera premisa que debemos establecer es que, en educación, siempre la necesidad de mejorar los procesos - y con ello alcanzar los aprendizajeses una cuestión que puede suceder tanto en el aula como fuera de ella. En segundo lugar, la corrección del proceso dependerá de los medios implementados para dicho fin. A partir de esto, proponemos que por medio de metodologías activas participativas, como el aprendizaje basado en investigación (ABI), es posible incrementar y potenciar habilidades que contribuyan a la competencia genérica a alcanzar, como es el perfil de egreso, tanto en un contexto curricular o extracurricular; este último, evidenciado como parte de la experiencia que se quiere compartir en estas líneas y aceptando la idea de que las aulas y otros lugares pueden constituirse en espacios de aprendizaje como factor de construcción del conocimiento (Gómez Francisco y Rubio González, 2017).

\section{Hacia un lenguaje común y coherente que construya realidad}

La labor que ha desarrollado durante los últimos quince años la doctrina preocupada de la enseñanza del derecho ha permitido obtener claras luces de la problemática que enfrenta y, a su vez, plantear algunas soluciones, que en gran medida han sido consideradas por una parte de los involucrados. Me refiero a un acotado número de docentes que invierten tiempo en preparar los aprendizajes y que efectivamente direccionan su actuar de acuerdo con las necesidades reales de la educación del derecho (Coloma Correa, 2005: 168).

Cuando se hace referencia a la «enseñanza del derecho», lo primero que denota la expresión es un paradigma centrado en el docente y su cátedra magistral, en el que florecen aquellos fantasmas descritos supra, debido a la omisión principal del aprendizaje (Elgueta y Palma, 2015: 34), y denota los límites claros de lo que debe ser un aula de Derecho. Como señalan Elgueta y Palma (2015: 34): «Aparece solo el que enseña, quedando invisibilizado el que aprende. Se mantiene el foco en el profesor [...] el alumno es enseñado, no participa como sujeto activo». De esta manera, el real problema en las escuelas de Derecho parte de la poca comprensión y aceptación del paradigma en que el docente deja de ser el protagonista para convertirse en un mediador.

$\mathrm{Si}$ aceptamos que el lenguaje crea o configura realidades, entonces debemos aceptar que un primer paso en la tarea de solucionar los obstáculos en la educación del derecho es precisamente comenzar por determinar la conceptualización correcta y el sentido que debemos darle al proceso educativo. El enfoque debe centrarse en un proceso dominado por el aprendizaje bajo el mecanismo de una enseñanza centrada en el alumno, como establece Rué (2007: 56) cuando señala que «se observa que el aprendizaje centrado en el alumno es de un mayor nivel de compromiso y trabajo del estudiante, favoreciendo la autonomía y generando competencias para el aprender a aprender en colaboración con los compañeros». 
Un lenguaje uniforme en torno al proceso de aprendizaje y enseñanza es el paso inicial para que el cambio tenga un sentido desde el lenguaje, adaptando con ello la realidad práctica de cada docente al interior de la sala de clases y bajo el contexto del desarrollo de habilidades y competencias que tributen al perfil de egreso. Desde el lenguaje común, la práctica debiese transformarse de manera coherente con lo que dicta el nuevo y real enfoque, hasta abandonar el enfoque centrado en la enseñanza informativa, pasiva y teórica, por una enseñanza formativa, receptiva, activo-participativa y práctica (Flores, 2014: 123).

\section{Explorando un camino en la educación del derecho acorde con las necesidades del siglo XXI}

La enseñanza del derecho debe concebirse respecto del aprendizaje, en cuanto éste lo precede en orden e importancia; solo de esta manera es posible entender el real alcance del proceso de aprendizaje y enseñanza, como señalan Elgueta y Palma (2015: 40): «Como un proceso de interpretación y construcción del conocimiento, acumulativo, autorregulado, orientado por algún objetivo».

Para obtener resultados a través de los nuevos paradigmas en educación, Flores (2014: 124) destaca que «es necesario implementar otros métodos en la enseñanza del derecho, más allá de la tradicional cátedra o lección magistral», para asegurar de forma efectiva que el aprendizaje sea alcanzado. Ha quedado claro entonces - después de lo comentado por los autores- que la enseñanza tradicional es un obstáculo en el desarrollo de habilidades de mayor complejidad que permitan acreditar competencias necesarias para la vida laboral.

Debemos aceptar que en la educación del derecho $-\mathrm{y}$ en general en la educación superior de nuestro país, estemos más o menos de acuerdo en este punto- se preceptúa como enfoque educativo el aprendizaje por competencias (Aedo Barrena, 2014: 105) o basado en competencias como fórmula para alcanzar los aprendizajes en un contexto actual (De Miguel, 2006: 56). Este modelo posee críticos que niegan su eficacia y hasta su naturaleza (Díaz-Barriga, 2006: 8), pero, más allá del campo de la discusión de los teóricos en educación sobre la naturaleza de modelo o enfoque, lo cierto es que el perfil de egreso de cada carrera universitaria da cuenta del modelo por competencias que direcciona el quehacer educativo de las escuelas, lo que requiere centrar la mirada en el siguiente paso (Asún, Zúñiga y Ayala, 2013: 279): en los mecanismos, caminos o formas de alcanzar los logros de aprendizajes. Por consiguiente, tanto la metodología como el método están centrados en responder cómo el docente instala en sus estudiantes las habilidades requeridas (Coloma Correa, 2006: 141).

En palabras de Lazo (2011: 257):

Despejada la definición de la modalidad, comparece a continuación el de la elección de los métodos enseñanza, los que tienden a insertarse en la primera, una vez 
que existe claridad acerca de la competencia a desarrollar y su nivel de desarrollo. Los métodos de enseñanza que suelen identificarse como prácticas habituales son, genéricamente, los siguientes: lección magistral, estudio de casos, resolución de problemas, aprendizaje basado en problemas, aprendizaje basado en proyectos, aprendizaje cooperativo y contrato de aprendizaje.

Estas prácticas se manifiestan y dan cuenta en el lenguaje pedagógico de medios propios de las metodologías activas para cambiar tanto el enfoque como las formas en la práctica educativa, según señalan Silva Quiroz y Maturana Castillo (2017: 122):

Por metodologías activas entendemos aquellos métodos, técnicas y estrategias que utiliza el docente para convertir el proceso de enseñanza en actividades que fomenten la participación activa del estudiante y lleven al aprendizaje. Corresponden a aquellas metodologías que materializan este cambio en la forma de entender el aprendizaje, ya que se centran en las actividades más que en los contenidos, lo que implica cambios profundos en el actuar de profesores y estudiantes. Esto implica modificar la planificación de las asignaturas, el desarrollo de las actividades formativas y la evaluación de los aprendizajes, de manera tal que se promueva el alineamiento constructivo.

Además de entenderlo como un requerimiento cuyo eje articulador lo componen los métodos recurrentes que el docente pone a disposición del aprendizaje.

Se extrañan en la práctica pedagógica en derecho o en general en educación (Ruiz, 2008: 113) estrategias metodológicas basadas en investigación, o siquiera la sola práctica de ésta como técnica o ejercicio académico (González, 2003: 301) que den cuenta de una línea de escuela que articule el perfil de egreso con algunas asignaturas que tributen habilidades para la investigación y metodología investigativa, de manera que aporten directamente a la formación considerando evaluaciones del proceso en general. Esto permitiría que en cursos finales de la malla estas competencias puedan manifestarse en una investigación que reúna los requerimientos y cualidades de un trabajo científico y serio, que constituya un real aporte al conocimiento de la disciplina. Una clara exposición de la práctica y fomento de la investigación en pregrado en la realidad externa lo comenta González (2003: 301) al reseñar que

en el caso chileno, la mayoría de las universidades llevan adelante la enseñanza en base a clases magistrales donde los cursos de investigación son escasos [...] en las escuelas del sistema Common Law suelen incentivarse el desarrollo de las capacidades investigativas [...] además, durante la carrera se imparten una serie de cursos que se encargan de desarrollar la metodología de la investigación.

Según lo anterior, plantear el aprendizaje basado en investigación como estrategia metodológica dentro de la planificación constituiría claramente una labor de transformación de parte de las escuelas, que implicaría: i) un cambio curricular general orien- 
tado al desarrollo de habilidades en investigación; ii) la identificación de asignaturas que de manera indirecta puedan contribuir con habilidades que permitan desarrollar competencias en investigación, y su articulación con aquellas asignaturas propias de la metodología de la investigación; iii) adecuar la malla curricular implementando e incrementando asignaturas relacionadas en forma directa con el perfil de las habilidades en investigación; y iv) convencer a los docentes - sin impactar en la libertad de cátedra - a que dentro de sus asignaturas puedan contribuir con la aplicación de la investigación como método pedagógico, mediante trabajos de investigación que den cuenta de un proceso y un producto final de rasgos más científicos, que permitan a su vez complementar la teoría y la práctica, como expresan Piazza y Volpato (2016: 164):

La investigación como tal debe insertarse en el proceso de enseñanza-aprendizaje, proporcionando una combinación de teoría y práctica para construir nuevos conocimientos y, por lo tanto, la investigación no puede restringirse a un momento específico como la monografía o un trabajo de conclusión del curso (la traducción es nuestra).

La realidad de las escuelas de Derecho en general dicta por ahora una imposibilidad concreta de concebir el aprendizaje basado en investigación como estrategia metodológica, y que por lo demás desborda la intención de este trabajo. Ello resulta evidenciable, por un lado, por las propias mallas de carrera; luego, por la escasa práctica del aprendizaje basado en investigación como método pedagógico; y, por último, por el escaso interés en general por investigar, lo que en pregrado sucede tanto a estudiantes como docentes, estos últimos que debieran ser encargados de promoverlo e instalarlo de manera adecuado (Montero, 2009: 19). Una idea similar plantea Cicero (2018) cuando señala que la adecuación curricular no solo incumbe a la modificación de las mallas, sino también la modificación en la manera de enseñar y las formas de concebir el aprendizaje.

\section{El aprendizaje basado en investigación: Una propuesta coherente}

Sin ánimo de escribir un ensayo sobre el aprendizaje basado en la investigación, resulta necesario, sin embargo, reservar algunas líneas para su tratamiento, desde donde se podrá deslindar algunas ideas en torno a la investigación como estrategia o como método pedagógico.

La preocupación por una educación en que la experimentación o investigación sean herramientas pedagógicas en el aprendizaje se remontan al año 1914, cuando el pedagogo norteamericano John Dewey fundamentó que la educación tiene que entregar al que estudia herramientas para la vida a través de la experiencia, la que debe ser recorrida por medio de la exploración guiada por el método científico (Colom, Bernabéu y Domínguez, 2002). A su vez, Ernest Boyer, también profesor norteame- 
ricano, señala que en la educación universitaria faltaban oportunidades de participación para los estudiantes en actividades relacionadas con la investigación y, en consecuencia, carecían de habilidades relacionadas con ella (Torres Tobías, 2010: 15). Así describían en igual sentido los problemas que la educación en general enfrentaba en Estados Unidos, y en particular en la educación universitaria a partir de la segunda mitad del siglo XX.

Con el tiempo, esta preocupación fue suscitando cada vez más interés en Estados Unidos, ya en 1998 se reunió la Comisión Boyer para la educación universitaria, encabezada por Boyer, entonces presidente de la Fundación Carnegie para el avance de la enseñanza, e integrada por una docena de académicos, intelectuales y científicos distinguidos. La Comisión estableció que el problema de estas instituciones radicaba en el bajo compromiso de creación y producción del conocimiento, que separaba las actividades pedagógicas de la investigación en las aulas universitarias (Rodríguez Gómez, 2000: 78). Esto dio origen a que algunas instituciones de educación superior comenzaran a centrar su enfoque en la investigación, a través de lo que llamaron «nueva estrategia didáctica», la que sería conocida desde ese momento como aprendizaje basado en investigación (Peñaherrera, Chiluiza y Ortiz, 2014: 206).

En el mismo sentido, pero en contexto latinoamericano, el Tecnológico de Monterrey en México ha incorporado desde 2010 el aprendizaje basado en investigación como estrategia metodológica, con el objetivo de conectar la investigación con la enseñanza, lo que ha significado que sea concebido hoy no solo como un enfoque, sino como una herramienta variada tendiente a fortalecer el aprendizaje y la enseñanza (Espinel y otros, 2016: 50).

El aprendizaje basado en investigación, sea como enfoque, estrategia o método pedagógico, puede ser considerado como una referencia de innovación educativa, que tras su aplicación se ha complementado y nutrido de algunas teorías educativas, como la teoría constructivista de Piaget, el aprendizaje significativo de Ausubel e inclusive la teoría social de Vygotski (Peñaherrera, Chiluiza y Ortiz, 2014: 207).

Adelantándonos a algunos cuestionamientos que podrían emerger, es importante hacer referencia a que los problemas descritos en la educación universitaria, tanto de Estados Unidos como de México, no son fenómenos aislados o propios de sus sociedades. Por el contrario, dichos problemas se replican en nuestro sistema de educación superior con mayores o menores matices, teniendo en cuenta su clara manifestación como problemática en nuestras escuelas de Derecho. En este sentido, el aprendizaje basado en investigación se propone como una de las tantas soluciones que pueden replicarse en nuestras aulas.

Para efectos de este trabajo, debemos hacer otra aclaración, y es que nuestra pretensión no es instalar el aprendizaje basado en investigación como estrategia metodológica en nuestras escuelas, puesto que ello significaría, como se señaló, una reestructuración curricular que merece más estudio y análisis. 
Un primer cuestionamiento es determinar qué ventajas o beneficios ofrece la investigación en el contexto educativo en general. De acuerdo con David Mora (2006: 15), del Instituto Internacional de Integración, la investigación en el proceso educativo «no es más que recuperar o reestablecer el vínculo natural entre los seres humanos y sus mundos socionaturales», lo que reconoce la virtud original de la indagación y exploración como fórmula para conocer el mundo que lo rodea o explicar aquello que desconoce.

La investigación permite asociar en el proceso educativo la teoría y la práctica (Mora, 2009: 35), cuestión que en derecho ha sido parte del problema, sobre todo cuando se ha descrito la doble dimensión entre estudiante y abogado, principalmente por falencias en la coordinación y formación entre ambos al interior de las escuelas. Además, la investigación por parte del estudiante constituye una oportunidad para el análisis, el ensayo, la reflexión, la innovación y la disciplina académica (Rivadeneira y Silva, 2017: 7), esta última muchas veces confundida con la memorización de conceptos y contenidos, como en la vieja escuela; habilidad que claramente puede ser incorporada en el estudiante a través de la investigación, de modo de mantener el estándar de rigurosidad que tanto buscan las escuelas de Derecho en sus alumnos.

De acuerdo con Álvarez, Orozco y Gutiérrez (2011: 64), las competencias investigativas «i) consolidan conocimientos; ii) ayudan a generar aportes significativos; iii) desarrollan habilidades y destrezas; iv) mejoran la práctica profesional», con lo que destacan una serie de dimensiones de desarrollo que la investigación como antecedente aporta al proceso educativo. Frente a esto, nos hacemos la interrogante: $i s o n$ las habilidades que desarrolla la investigación las que interesan en la educación del derecho?

La respuesta no es sencilla si intentamos responderla desde la diversificación de las disciplinas. Sin embargo, parece más auspiciosa si aceptamos que el desarrollo de las habilidades en cuestión son transversales a cualquier disciplina, y que al interior de cada una los contenidos de los aprendizajes esperados entregan el material específico que se complementa con las destrezas y habilidades proporcionadas por la investigación, lo que permite generar competencias robustas desde la disciplina y desde las herramientas generales que aporta el aprendizaje basado en investigación (Pozuelos, Rodríguez y Trave, 2012: 563).

Las habilidades que desarrolla la investigación en los estudiantes como herramienta metodológica se centran en cualidades y competencias que cualquier profesional debe reunir mientras adquieren las capacidades de interpretación, análisis y síntesis, todas estas de orden superior en la taxonomía de Bloom y Anderson, habilidades que obligan al estudiante de Derecho a ir más allá de la memorización y lo trasladan al campo del entendimiento profundo. Se relaciona además con la formación de la investigación la síntesis de información, tan necesaria en la labor de los abogados como la redacción de los hechos en un escrito, en orden a tareas más simples, o en 
tareas de mayor complejidad, como expresan Viteri Briones y Vásquez Cedeño (2016: 40): «La investigación desarrolla en los estudiantes capacidades de [...] búsqueda de problemas no resueltos, el pensamiento crítico y otras capacidades como la observación, descripción y comparación; todas directamente relacionadas con la formación para la investigación».

De una u otra forma, tanto el estudiante de Derecho como el abogado en su práctica habitual se ven enfrentados a situaciones muchas veces complejas, que se espera puedan resolver identificando primero el problema, y buscando luego una solución real, adecuada y muchas veces creativa a la problemática que enfrentan.

Incentivar la investigación en los estudiantes favorece la formación de una cultura investigativa y una herramienta para descubrir y dar solución a todo tipo de problemas. Forja en ellos un carácter reflexivo, crítico y constructivo como sujetos proactivos, como agentes transformadores de la sociedad que le toca vivir (Viteri Briones y Vásquez Cedeño, 2016: 40).

Explorando las ideas del pensamiento de Lipman (2011: 87), el aprendizaje basado en la investigación se presenta como una oportunidad que, por medio de la investigación y sobre todo la cultura investigativa entre los alumnos, permite alcanzar niveles elevados de pensamiento:

El pensamiento de orden superior implica tanto un pensamiento creativo como crítico; la comunidad de investigación, en la que los miembros se escuchan unos a otros con respeto, construyen ideas sobre los demás, se retan recíprocamente para reforzar sus argumentos, lo que ayuda a procesos inferenciales a partir de los que se afirme; el dialogo sucede cuando cada uno de los participantes tiene en mente el otro o los otros; los criterios que se desarrollan son parte de razones de una fiabilidad muy estricta.

El estudiante que se inserta en una comunidad investigativa resalta y refuerza las virtudes que el trabajo colaborativo y responsable aportan al estudiante como sujeto que aprende y se forma. Con ello, logra que el enfoque de aprendizaje basado en investigación desarrolle valores del investigador en los estudiantes.

A modo general e ilustrativo, hemos seguido el siguiente ejercicio. De las 29 universidades que hemos tenido a la vista para extraer estadísticas de sus mallas curriculares, hemos sintetizado las competencias que más se repiten en sus perfiles de egreso, según las habilidades propias de la argumentación e investigación jurídica. Para ello, recopilamos la información a partir de la observación directa de cada una de las mallas ubicadas en los sitios web de las universidades. Luego, se estableció un descriptor base, que corresponde al perfil de egreso de Derecho de la Universidad Santo Tomás, a partir del cual se fueron cotejando las habilidades descritas en los restantes perfiles de egreso. El resultado fue un ejercicio de inclusión de habilidades según el número acumulado de habilidades, mientras que se excluyeron las habilidades que se reite- 
raban menos. Esto dio cuenta de un perfil de egreso consolidado o genérico, el que se puede estructurar en las siguientes competencias: evaluar situaciones jurídicas e identificar problemas y fenómenos normativos resolviéndolos de manera oral y escrita, a partir del diseño de estrategias de solución de problemas desde el razonamiento, la argumentación y un adecuado manejo de fuentes. Esta descripción constituye un punto de partida, como competencia generalísima relacionada con las diferentes habilidades desarrolladas a través de la investigación. De esta manera, las habilidades de razonamiento, argumentación, resolución creativa de problemas, análisis crítico de situaciones y otras descritas se entienden por completo subsumibles en una competencia del perfil de egreso genérico a las que puede contribuir el aprendizaje basado en la investigación (Martínez y Buendía, 2005). Esto contribuye de manera directa en la labor del abogado, en la que la investigación no solo constituye la base del ejercicio de la profesión (López, 2011: 233), sino que sirve de guía para interpretar y aplicar una o más normas jurídicas, y fomenta una de las competencias que permiten al abogado del siglo XXI mediar conflictos, analizarlos e innovar en soluciones (Benfeld, 2016: 149).

\section{Propuesta de metodología didáctica}

\section{Experiencia pedagógica extracurricular a partir} del aprendizaje basado en la investigación

Frente a la necesidad de crear una cultura de investigación en nuestra escuela, y teniendo en consideración las habilidades desarrolladas a través de la investigación, las cuales tributan directamente al perfil de egreso, es que, sin otro propósito que dar a conocer nuestra práctica, se presenta la experiencia que en la Escuela de Derecho de la Universidad Santo Tomás sede Antofagasta se ha estado implementando con tibieza desde el año 2017, y con más estudiantes durante 2018 y 2019. Esta experiencia se ha visto interrumpida por los eventos ocurridos a partir de octubre de 2019, que han imposibilitado el cierre normal del ciclo anterior y el comienzo previsto para el año 2020.

El taller de investigación y debate se constituye como una iniciativa docente a través de la cual un grupo inicial de cinco estudiantes y un académico de la Escuela, quienes, tras la preparación para un torneo de debate a nivel nacional, comenzaron a evidenciar deficiencias en el proceso de preparación, como la búsqueda y selección de información bibliográfica, los pasos para generar información objetiva, la elaboración y articulación de argumentos, entre otros.

Por ello, a partir de mayo de 2017 se decidió implementar un taller abierto para todos los estudiantes de la carrera de Derecho de la Universidad Santo Tomás sede Antofagasta. La invitación fue extensiva para alumnos de primer a quinto año. Comenzó con reuniones semanales los viernes, para compartir información jurídica, y discutir 
ideas y proyectos, con la idea de comenzar a investigar temas de relevancia jurídica e interés propio de cada alumno. Durante el primer año, la actividad principal estuvo centrada en articular entre el pequeño grupo de estudiantes algunos talleres de metodología de la investigación y argumentación jurídica. El segundo año, en 2018, el número de integrantes se incrementó de manera importante, lo que significó comenzar a organizar nuevos talleres y una articulación entre los asistentes de los distintos niveles al taller. En este sentido, se evidenció que las conductas de entrada al taller eran diversas, por lo que las dificultades resaltaban por sobre las competencias en el ámbito de la investigación. Por ello, se comenzó a desarrollar un trabajo diferenciado entre los de mayor y menor avance.

Así, en 2018 los participantes expusieron los resultados de sus investigaciones en un seminario interno celebrado en noviembre. Esto se tradujo en un escenario propuesto y concretado por los propios estudiantes a partir del patrocinio de la Escuela y la dirección de un docente de planta, la participación en concursos internos de la Dirección de Asuntos Estudiantiles (DAE) para obtener los recursos y financiar el encuentro.

El tercer año congregó también a un importante número de estudiantes que participaron en forma activa durante el año, asistiendo a los talleres y a todas las jornadas de trabajo, con la única excepción que significó la imposibilidad de hacer el seminario en la fecha agendada.

El taller de investigación se implementa y se mantiene en los años sucesivos, frente a la poca cultura de investigación, que se manifiesta tanto en las mallas como por la propia práctica docente en las distintas asignaturas. En tanto, los estudiantes han encontrado en el taller de investigación y debate, que los reúne cada semana, el espacio para investigar, discutir, innovar y emprender en torno a la indagación y el interés por desarrollar nuevas habilidades y conocimientos. Todo ello se ha manifestado en que el equipo se ha incrementado en número cada año, sobre todo con alumnos de primer año, desde donde entendemos - según nuestra apreciación como formación primaria- debe partir la base en investigación jurídica, práctica que fomente una cultura pedagógica investigativa que permita de paso desarrollar hábitos de indagación y resolución de problemas, tributando directa e intencionadamente a las competencias del perfil de egreso (Viteri Briones y Vásquez Cedeño, 2016: 40).

Entendemos que el taller de investigación se traduce en un aprendizaje eficiente para el estudiante, en el que relaciona su propia actividad con situaciones o experiencias positivas para él (Mora, 2009: 53), y el producto de la construcción que conlleva la investigación se transforma para la comunidad en un reporte de su actividad indagadora (Martínez y Buendía, 2005: 16).

El contacto directo y personal con los estudiantes permitió identificar un sinnúmero de necesidades y falencias de manera transversal, sin distinción de cursos, como conducta de entrada al taller. Estas necesidades se manifiestan en habilidades 
de base, como verificar que había estudiantes con problemas de lectoescritura, en especial de primer año; algunos con evidentes falencias para comprender lo que leen; otros con manifiestos problemas para redactar las ideas en una papel; además, fue posible evidenciar estudiantes con serios problemas en el manejo de Word, lo que a todas luces se trasformó en una primera tarea en abordar, a través de sesiones particulares de ejercitación en lectura y escritura a nivel general. Esta es una situación emergente y no prevista a la hora de planificar el taller, por lo que no está incorporada en la planificación y el plan inicial, pero que sin duda se manifiesta como una necesidad de la que debemos ocuparnos a través de otras instancias formativas.

Por otro lado, resultó evidente encontrarnos con un escaso conocimiento en metodología para investigar en buena parte de los estudiantes asistentes, por lo que en el tiempo de vida del taller se implementaron cuatro módulos básicos por año de metodología de investigación jurídica, como base conceptual para iniciar el trabajo de indagación. Con ello buscamos la adquisición por parte de los estudiantes de técnicas básicas que les permitieran iniciar el proceso en cuestión, además de incorporar algunas herramientas para identificar problemas a investigar y, en estudiantes de niveles avanzados, problemas o fenómenos de relevancia jurídica.

De esta forma, se comenzó a trabajar sistemáticamente con los estudiantes para lograr los objetivos trazados para cada periodo y verificables de manera directa e inmediata, esto es, la generación de una pequeña investigación que asegure la participación de cada estudiante en el seminario interno de la Escuela de Derecho sede Antofagasta, organizado por los estudiantes del taller, el centro de alumnos de la carrera y el docente a cargo del taller.

A partir de la escasa experiencia en lo temporal, pero cuantiosa en lo esencial, hemos construido como taller de investigación y debate una estrategia metodológica extracurricular, el que da cuenta de la implementación del aprendizaje basado en la investigación como método didáctico central al interior del taller, y cuyo objetivo primordial es instalar la cultura investigativa como uno de los motores de aprendizaje de nuestros estudiantes.

Frente al cuestionamiento de si es posible aplicar el aprendizaje basado en la investigación como estrategia metodológica en el taller o fuera del aula, nuestra primera respuesta es que una vez que el taller centra su quehacer en los medios más que en el resultado final, da cuenta de que en las etapas intermedias del proceso de investigación se potencian y desarrollan las habilidades que interesan. A partir de esta convicción y práctica es que el taller sustenta su quehacer a través del aprendizaje basado en la investigación como estrategia metodológica. Lo segundo es que no necesariamente se debe encontrar en un contexto de aula y currículo establecido para poner en práctica la metodología; por el contrario, aceptando el currículo adaptado, es posible concebirlo como parte integrante de la formación del estudiante y, por tanto, apto para aplicar esta estrategia metodológica. 
Tabla 1. Plan de acción del taller de investigación y debate extracurricular, carrera de Derecho UST, Antofagasta

\begin{tabular}{|c|c|c|}
\hline Objetivos & Acciones & Medios \\
\hline $\begin{array}{l}\text { 1. Adquirir métodos, técnicas y } \\
\text { herramientas metodológicas de } \\
\text { investigación jurídica por parte de } \\
\text { los estudiantes asistentes (estudian- } \\
\text { tes). }\end{array}$ & $\begin{array}{l}\text { - Desarrollar en alumnos las habilidades investiga- } \\
\text { tivas. } \\
\text { - Diseñar e implementar talleres y cursos sobre } \\
\text { metodología de investigación jurídica. } \\
\text { - Diseño de planes de seguimiento. }\end{array}$ & - Talleres, cursos y charlas. \\
\hline $\begin{array}{l}\text { 2. Incentivar a estudiantes a vincu- } \\
\text { larse en proyectos de investigación } \\
\text { internos de la universidad (dirección } \\
\text { del taller). }\end{array}$ & $\begin{array}{l}\text { - Difusión de programas y concursos de investigación } \\
\text { para estudiantes. } \\
\text { - Taller de preparación de proyectos. }\end{array}$ & $\begin{array}{l}\text { - Cursos de preparación proyectos. } \\
\text { - Acompañamiento en elaboración } \\
\text { de proyectos. } \\
\text { - Difusión de concursos internos. }\end{array}$ \\
\hline $\begin{array}{l}\text { 3. Desarrollar tres niveles de investi- } \\
\text { gación jurídica asociados a criterios, } \\
\text { junto a las habilidades propias de la } \\
\text { investigación (sistema de terciles). }\end{array}$ & $\begin{array}{l}\text { - Según criterio de complejidad: profundización, } \\
\text { paralelos, comentarios, resolución de problemas, } \\
\text { etcétera. } \\
\text { - Según niveles: primer tercil (primer y segundo año); } \\
\text { segundo tercil (tercer y cuarto año) y tercer tercill } \\
\text { (quinto año). }\end{array}$ & $\begin{array}{l}\text { - Manejo interno de la dirección } \\
\text { del taller. }\end{array}$ \\
\hline $\begin{array}{l}\text { 4. Fomentar una cultura investigati- } \\
\text { va en los estudiantes participantes. }\end{array}$ & $\begin{array}{l}\text { - Incentivo y motivación de estudiantes. } \\
\text { - Organización de instancias para presentar resultados. } \\
\text { - Asistencia a seminarios, congresos, jornadas, etcéte- } \\
\text { ra, tantos internos como externos. }\end{array}$ & $\begin{array}{l}\text { - Incentivo directo para participar. } \\
\text { - Concursos internos de fondos } \\
\text { para asistencia a actividades } \\
\text { externas. }\end{array}$ \\
\hline $\begin{array}{l}\text { 5. Fomentar valores y principios del } \\
\text { investigador. }\end{array}$ & $\begin{array}{l}\text { - Objetividad. } \\
\text { - Respeto a la evidencia. } \\
\text { - Tolerancia. } \\
\text { - Honestidad. } \\
\text { - Rigor. }\end{array}$ & $\begin{array}{l}\text { - Durante el desarrollo del taller. } \\
\text { - Observación directa. }\end{array}$ \\
\hline
\end{tabular}

Lo anterior se manifiesta en el plan que hemos fijado para el taller en general, el que se ha implementado en los años de funcionamiento que esperamos seguir aplicando en los años venideros, como muestra la tabla 1.

\section{Desarrollo y fortalecimiento de competencias a través del taller de investigación}

A la hora de buscar el punto de inicio en la formación de una cultura investigadora en los estudiantes de Derecho, lo que debemos considerar es que, en el primer año de gran parte de las carreras de la disciplina nos encontramos con alumnos con diversas destrezas y habilidades, dentro de los cuales ingresan incluso algunos con competencias descendidas, a los que se les denomina alumnos «desaventajados», para los que su tarea inicial y básica es fortalecer las competencias de lectoescritura y más (Coloma Correa y Agüero, 2012: 46). Es precisamente ante este panorama que la oportunidad de desarrollar una investigación formativa cobra vital importancia, pues la tarea se vuelca a trabajar las competencias puras que la indagación puede aportar. De nuevo, queremos tomar las ideas de Coloma Correa y Agüero (2012: 48), quienes a través de su artículo «Los abogados y las palabras» desarrollan un examen certero 
de lo que puede señalarse como el principio del fin, cuando aluden a la actividad del docente que tiende a pensar que las habilidades de lectoescritura se absorberán por la mera utilización de medios escritos a través de la imitación. Estas habilidades son imposibles de inculcar en los estudiantes si no se conciben nuevos métodos para fortalecerlas, como es el caso de la investigación, en la que el mismo autor va descubriendo y aprendiendo significativamente por medio de la escritura creativa, responsable y dirigida por parte del docente (Ruiz, 2008: 128).

En este sentido, la primera tarea ha sido evaluar y fortalecer las habilidades en lectura, tanto la comprensión, la síntesis y el análisis del texto, como la búsqueda de fuentes de información que usarán en sus investigaciones. Si bien estamos lejos de evaluar si la comprensión lectora ha mejorado en los estudiantes que participaron en el taller en este corto periodo, sí tenemos la seguridad de que el volumen de lecturas al que han sido sometidos ha sido abundante y variado, lo que asegura por lo menos una mayor dimensión de análisis y creatividad a la hora de elegir un tema de investigación y sin duda, como consecuencia natural, el comenzar a mejorar la escritura (Narváez Hernández, 2019: 86).

Aprender a investigar no es una tarea fácil. Por ello debe ponerse en práctica desde los primeros años, a fin de salvaguardar y eliminar las barreras o factores de tipo sociales, institucionales, pedagógicos y sicológicos (Rivadeneira y Silva, 2017: 9), los que muchas veces complejizan el acercamiento o el desarrollo de una práctica investigativa. En el caso de los estudiantes del taller, los temores iniciales se presentaron en dos sentidos: primero, el desconocimiento de la metodología y técnicas de investigación; segundo, la indecisión en la elección de temas a investigar, debido al pensamiento convergente con el que conviven los estudiantes de Derecho. En este sentido, el acercamiento a lecturas de diversos temas en el taller permitió a los alumnos ir ampliando el conocimiento del derecho, reconociendo además las técnicas y metodología en la investigación, lo que sin duda permitió ampliar su mirada e introducirlos hacia un pensamiento divergente, con el que a su vez incrementaron el horizonte temático.

El desarrollo de las competencias en investigación debe comenzar en los primeros años de formación del estudiante de Derecho. Tal vez el primer semestre pueda resultar algo apresurado e invasivo para el nuevo estudiante, pero a partir del segundo semestre, ciertamente debe producirse el contacto necesario con la investigación, con lo que se asegura que el trabajo a desarrollar sea sistemático, prolongado en el tiempo, y se logre la obtención de habilidades propias de la investigación desde las más simples hasta las más complejas en los semestres sucesivos. Esta base busca automatizar las habilidades del estudiante y asegurar el salto en el dominio de otras competencias de mayor complejidad. Es precisamente lo que busca el taller: lograr en nuestros estudiantes, una vez finalizado el proceso de participación desde primer año hasta su egreso, la adquisición de habilidades tributadas desde la investigación, 
que permitan el avance progresivo y sistemático en los terciles propuestos. Resultados que creemos solo son verificables al cabo de un periodo mayor al que hasta ahora lleva el taller, puesto que el proceso y la sistematización desde la investigación como metodología y el paso a convertirse en un verdadero instrumento requiere de tiempo, dedicación y esfuerzo.

El desarrollo de habilidades de carácter formativo en investigación durante los primeros años posibilitará el constante perfeccionamiento, a través de niveles de sistematicidad de las herramientas metodológicas, que permitan a su vez ir escalando en complejidad (Viteri Briones y Vásquez Cedeño, 2016: 40) tanto el objeto de la investigación como la modalidad. La primera etapa en el proceso de investigación debe tener como conductor a un docente que permita desarrollar en los estudiantes una estructura lógico-metodológica del proceso investigativo (Restrepo, 2006), lo que debe entenderse como las bases conceptuales y esenciales. Estructuras que tienen una clara articulación con la asignatura de argumentación jurídica que dictan algunas escuelas de Derecho y desde donde es posible aportar al desarrollo de la lógica, el razonamiento deductivo e inductivo. A medida que el proceso avanza, el mismo docente debe morigerar su intervención directa dejando el rol conductor, para pasar a ser un facilitador cuando el estudiante manifieste avances y aprendizaje en la técnica y el método.

\section{Articulación según terciles}

En el taller de investigación jurídica y debate de la Escuela, y reconociendo el carácter de sistematicidad, progresión y complejidad, se propone el trabajo a partir de terciles organizados por año académico, con modalidades de investigación desde actividades simples y formativas a investigaciones de mayor complejidad, como un comentario de sentencia, una investigación doctrinal o aplicada.

El primer tercil agrupa a alumnos de primer y segundo año. Incluye formación inicial y básica de habilidades de investigación, acciones metodológicas tendientes a fortalecer lectoescritura, y la investigación bibliográfica como parte esencial del aprendizaje (Ruiz Hoyos y Cardona Rivas, 2016), además de la identificación de problemas sencillos y temáticas de interés particular en el área jurídica. La modalidad de investigación es la profundización de temas revisados en algunas asignaturas de los primeros semestres, como establecer paralelos entre el origen y la actualidad de una institución jurídica; por ejemplo, el paralelo entre Roma y el Código Civil respecto de la tradición.

El segundo tercil agrupa alumnos de tercer año y cuarto año. Incluye formación intermedia, en que las herramientas metodológicas ya se encuentran instaladas, con manejo de mayor cantidad y calidad de fuentes. Considera identificación de fenómenos o problemas normativos de mayor complejidad y la solución de éstos, con el 
desarrollo de habilidades instaladas de aplicación, síntesis y evaluación en torno a una investigación. La modalidad de investigación puede ser una profundización de discusión doctrinal, una monografía o la solución de un problema jurídico simple, o la confección de instrumentos para obtener información como base estadística o para la investigación. Se concentra en este tercil la participación en concursos de proyectos y participación en seminarios externos.

El tercer tercil agrupa a estudiantes de quinto año, cuyo perfil se orienta a reconocer problemas jurídicos de mayor complejidad. La modalidad de investigación se orienta a la investigación aplicada, dentro de la cual es más recurrente el comentario de sentencias que se reúnen en un portafolio, la ayudantía en alguna investigación docente, participación en seminarios externos, etcétera. Se complementa con una clínica jurídica que se dicta en el noveno y décimo semestres.

La estructura anterior es el fruto de la experiencia acumulada durante estos escasos años en que se ha implementado el taller, del que se han obtenido algunas ideas respecto a prácticas que se están siguiendo y otras que se espera sean desarrolladas en lo sucesivo, lo que no significa que esta propuesta sea conocimiento asentado respecto de la práctica y aplicación del aprendizaje basado en la investigación de manera extracurricular. Por el contrario, reconocemos la necesidad de seguir avanzando en el desarrollo de las habilidades investigativas de los estudiantes de Derecho y bajo una apertura total respecto de aportes desde otras escuelas para perfeccionar nuestra estrategia pedagógica libre, voluntaria y espontánea.

\section{Conclusiones}

A partir de la experiencia y en una primera aproximación, entendemos que se desprenden, más que resultados evidenciables, algunas conclusiones en el corto plazo, que apuntan a considerar el aprendizaje basado en la investigación como una alternativa interesante dentro del proceso de aprendizaje y enseñanza del derecho. La implementación de la investigación no queda condicionada solo a la ocurrencia de asignaturas que la incorporen como parte medular de la malla académica, sino como una alternativa al interior de cada practica pedagógica o a través de experiencias extracurriculares, que permitan en definitiva fortalecer aquellas habilidades que la práctica común y recurrente de las formas clásicas no logra desarrollar en su plenitud.

Es posible lograr la adquisición y evolución de habilidades que tributen al perfil de egreso, tanto al interior del aula como fuera de ésta, a través de experiencias que, si bien están fuera del currículo, son perfectamente compatibles y coadyuvantes.

Se hace necesario comprender, compartir y difundir la necesidad de un desarrollo temprano de las habilidades investigativas en alumnos de primer año en adelante. A partir del positivo efecto que genera en los estudiantes, se traduce en un mayor inte- 
rés y motivación no solo por la carrera, sino también por la construcción de su propio conocimiento a lo largo de los años de estudio.

Los efectos a largo plazo estarían relacionados con las habilidades propias del perfil de egreso y del abogado de siglo XXI, como resolución de problemas, análisis crítico y toma de decisiones, las que reforzarán aún más su conducta de salida. Las habilidades de orden superior, que muchas veces no son alcanzadas por la clase y la evaluación tradicional, se logran ejecutar y ejercitar a partir de la práctica investigativa en un ambiente en que la cultura por la indagación es un hábito y no una obligación impuesta.

A través de nuestra experiencia, hemos logrado identificar de manera interna y en constante reconstrucción, distintos niveles de desarrollo en las habilidades e intereses de investigación, partiendo desde un nivel básico, como la profundización de un tema ya visto en clases, un contenido cercano y amigable del que se desea conocer más, en el caso de alumnos de niveles inferiores; un segundo nivel de investigación que busque dar respuestas teóricas a problemas identificados o a temas que requieran una profundidad dogmática mayor; para pasar a un tercer nivel en que los alumnos del nivel superior sean capaces de llevar a cabo investigaciones aplicadas, como un comentario de sentencia, la resolución de un caso difícil, en el que deben ir más allá de la norma para dar solución a su cuestionamiento o la resolución de un problema jurídico identificado por ellos, lo que tradicionalmente se resuelve a través de una tesis, tesina, seminario de grado o memoria según las distintas denominaciones que le dan las mallas de universidades chilenas, para que se complementen el conocimiento del derecho y las herramientas de la metodología jurídica.

Otra consideración que se debe tener en cuenta es el periodo que se requiere para lograr resultados visibles y evidenciables. Debe ser en un tiempo prolongado, puesto que las habilidades finales son consecuencia de un proceso formativo que en este caso acompaña al proceso curricular desde un costado. Por ello, la progresión es a su vez más lenta, a lo que se suman los obstáculos propios que tiene la investigación en cuanto a la práctica misma.

\section{Referencias}

Aedo Barrena, Cristian (2014). «Formación por competencias y la enseñanza del Derecho: Universidad de Chile». Revista Pedagogía Universitaria y Didáctica del Derecho, 1 (1): 104-113. DOI: 10.5354/0719-5885.2015.35966.

Álvarez, Víctor, Oilda Orozco y Antonio Gutiérrez (2011). «La formación de competencias investigativas profesionales: Una mirada desde las ciencias pedagógicas». Cuadernos de Educación y Desarrollo, 3 (24): 101-113. Disponible en https://bit. ly/zerbWpo. 
ANDERson, Lorin y David Krathworhl (2001). A taxonomy for learning, teaching and assessing: A revision of Bloom's taxonomy of educational objectives. Nueva York: Longman.

AsúN, Rodrigo, Claudia Zúñiga y María Ayala (2013). «La formación por competencias y los estudiantes: Confluencias y divergencias en la construcción del docente ideal». Revista Calidad de la Educación, 38: 277-304. DOI: 10.31619/caledu.n38.112.

BENFELD, Johann (2016). «La discusión sobre enseñanza del derecho en Chile dentro del nuevo paradigma universitario: Una tarea pendiente». Revista de Derecho (Coquimbo), 23 (1): 143-171. Disponible en https://bit.ly/316oOdV.

BöHMER, Martín (2009). La enseñanza del derecho y el ejercicio de la abogacía. Madrid: Gedisa.

Camilloni, Alicia (2019). «La enseñanza del derecho orientada al desarrollo de la creatividad». Revista Pedagogía Universitaria y Didáctica del Derecho, 6 (1): 5-22. DOI: 10.5354/0719-5885.2019.53743.

Cicero, Nidia Karina (2018). «Innovar la enseñanza del derecho: ¿Solo se trata de tecnologías de la información y comunicación?». Revista Pedagogía Universitaria y Didáctica del Derecho, 5 (2): 91-109. DOI: 10.5354/0719-5885.2018.51976.

Colom, Antoni, José Luis Bernabéu y Emilia Domínguez (coordinadores) (2002). Teorías e instituciones contemporáneas de la educación. Barcelona: Ariel.

Coloma Correa, Rodrigo (2005). «El ocaso del profesor Binns: Un ensayo acerca de la enseñanza del derecho en Chile». Ius et Praxis, 11 (1): 133-172. DOI: 10.4067/ So718-00122005000100006.

-. (2006). «Hacia una enseñanza del derecho efectiva». Revista Escuela de Derecho, 7 (7): 123-141. Disponible en https://bit.ly/3dmRSDh.

Coloma Correa, Rodrigo y Claudio Agüero (2012). «Los abogados y las palabras: Una propuesta para fortalecer competencias iniciales en los estudiantes de Derecho». Revista de Derecho (Coquimbo), 19 (1): 39-69. DOI: 10.4067/ So718-97532012000100003.

Cruz-Coke, Ricardo (2004). «Evolución delas universidadeschilenas, 1981-2004». Revista Médica Chilena, 132 (2):1.543-1.549. DOI:10.4067/So034-98872004001200014.

De Bianchetti, Alba, Susana Fridman e Hilda Zarate (2003). "La evaluación del proceso enseñanza-aprendizaje en el derecho». Comunicaciones Científicas y Tecnológicas, 2: 1-4.

De Miguel, Mario (2006). Modalidades de enseñanza centradas en el desarrollo de competencias: Orientaciones para promover el cambio metodológico en el espacio europeo de educación superior. Oviedo: Universidad de Oviedo.

DíAZ-BArRiga, Ángel (2006). «El enfoque de competencias en la educación: ¿Una alternativa o un disfraz de cambio?». Perfiles Educativos, 28 (111): 7-36. Disponible en http://ref.scielo.org/5yjrxb. 
Elgueta, María Francisca y Eric Palma (2015). «De la enseñanza del derecho a la didáctica general del derecho». Instituto de Investigación Jurídica, Universidad Autónoma de México. Disponible en https://bit.ly/3140tII.

EsPINEL, Johanna, Carmita Ramírez, Junes Robles y Richard Ramírez (2016). «Aprendizaje basado en la investigación». Revista Ciencia UNEMI, 9 (21): 49-57. DOI: 10.29076/issn.2528-7737volgiss21.2016pp49-57p.

Flores, Imer (2014). «Prometeo (des)encadenado: La enseñanza del derecho y los estudios de posgrado». Derecho y Cultura, 14 (15): 93-123. Disponible en https:// bit.ly/3hTJj6n.

Gómez Francisco, Taeli y Juan Rubio González (2017). «Cognición contextualizada: Una propuesta didáctica y psicopedagógica socioconstructivista para la enseñanza-aprendizaje del derecho». Revista Pedagogía Universitaria y Didáctica del Derecho, 4 (2): 40-63. DOI: 10.5354/0719-5885.2017.47970.

GonZÁLEZ, Felipe (2003). «Cultura judicial y enseñanza del derecho en Chile». Informes de Investigación, 14: 291-319. Disponible en https://bit.ly/3fPxjRO.

Lazo, Patricio (2011). «Formación jurídica, competencias y métodos de enseñanza: Premisas». Ius et Praxis, 17 (1): 249-262. DOI: 10.4067/So718-00122011000100011. Lipman, Matthew (2011). Pensamiento complejo y educación. Madrid: De la Torre.

LóPEZ, Sebastián (2011). «Para escribir una tesis jurídica: Técnicas de investigación en derecho». Ius et Praxis, 17 (1): 231-246. DOI: 10.4067/So718-00122011000100010.

Martínez, América y Agustín Buendía (2005). «Aprendizaje basado en investigación». Tecnológico de Monterrey. Disponible en https://bit.ly/316cltt.

Molina, Denyz (2009). «Repensar el perfil del abogado en un nuevo modelo de Estado social de derecho y de justicia». Revista Mexicana de Orientación Educativa, 6 (16): 8-15. Disponible en https://bit.ly/3fKF4bF.

Montero, Leidy (2009). «La formación para la investigación a nivel de pregrado». Tesis para obtener el grado de Maestra en Investigación Educativa, Magíster Universidad Autónoma de Yucatán.

Mora, David (2006). «Proceso de aprendizaje y enseñanza basado en la investigación». Integra Educativa, 2 (2): 13-82. Disponible en https://bit.ly/3hRVQY7.

-. (2009). «Pedagogía y didáctica crítica para una educación liberadora». Integra Educativa, (2) 1: 25-60. Disponible en https://bit.ly/3errIk6.

Narváez Hernández, José Ramón (2019). «Metodología crítica para la investigación científica del derecho». Revista Pedagogía Universitaria y Didáctica del Derecho, 6 (2): 81-96. DOI: 10.5354/0719-5885.2019.55309.

Peñaherrera, Mónica, Katherine Chiluiza y Ana Ortiz (2014). «Inclusión del aprendizaje basado en investigación (ABI) como práctica pedagógica en el diseño de programas de postgrados en Ecuador». Journal for Educators, Teachers and Trainers, 5 (2): 204-220. Disponible en https://bit.ly/315oDPV. 
PIAzza, Marcia y Gildo Volpato (2006). «Los núcleos de investigación en derecho y su impacto en la graduación». Revista Pedagogía Universitaria y Didáctica del Derecho, 3 (2): 159-171. DOI: 10.5354/0719-5885.2016.44666.

Pozuelos, Francisco, Francisco Rodríguez y Gabriel Trave (2012). «El enfoque interdisciplinar en la enseñanza universitaria y el aprendizaje basado en investigación: Un estudio de caso en el marco de la formación». Revista de Educación, 357: 561585. Disponible en https://bit.ly/3hUqFLE.

RESTREPO, Bernardo (2006). «Conceptos y aplicaciones de la investigación formativa, y criterios para evaluar la investigación científica en sentido estricto». Disponible en https://bit.ly/318jERE.

Rivadeneira, Elmina y Ricardo Silva (2017). "Aprendizaje basado en la investigación en el trabajo autónomo y en equipo». Negotium, 13 (38): 5-16. Disponible en https://bit.ly/3fNjyTt.

RODRíguez Gómez, Roberto (2000). «La reforma de la educación superior: Señas del debate internacional a fin de siglo». Revista Electrónica de Investigación Educativa, 2 (1): 68-88. Disponible en https://bit.ly/3fNjGSX.

RuÉ, Joan (2007). Enseñar en la universidad: El EEES como reto para la educación superior. Madrid: Narcea.

RuIz, Maite (2008). Evaluación de lengua escrita y dependencia de lo literal. Barcelona: Graó.

Ruiz Hoyos, Bayron y Dora Cardona Rivas (2016). «Propuesta de evaluación en el área básica del programa de Medicina de Universidad del Quindío 2008-2009 mediante la investigación-acción participativa». Revista Colombiana de Obstetricia y Ginecología, 67 (2): 89-100. DOI: 10.18597/rcog.378.

Silva Quiroz, Juan y Daniela Maturana Castillo (2017). «Una propuesta de modelo para introducir metodologías activas en educación superior». Innovación Educativa, 17 (73): 117-131. Disponible en http://ref.scielo.org/hjdy9y.

Solari, Enzo (2012). «El currículo chileno de estudios». Revista de Derecho de la Pontificia Universidad Católica de Valparaíso, 39 (2): 703-734. Disponible en https:// bit.ly/2Yqui4g.

Torres ToBíAs, Arturo (2012). «Aprendizaje basado en la investigación: Técnicas didácticas». Presentación del Programa de Desarrollo de Habilidades Docentes, Tecnológico de Monterrey. Disponible en https://bit.ly/2YpRwb1.

Viteri Briones, Telmo Alberto y Silvia Vásquez Cedeño (2016). «Formación de habilidades de investigación formativa en los estudiantes de la carrera de Ingeniería Comercial de la Facultad de Ciencias Administrativas de la Universidad de Guayaquil». Universidad y Sociedad, 8 (1): 36-44. Disponible en http://ref.scielo. org/7xtj4b. 
Zilberstein Toruncha, José y Silvia Olmedo Cruz (2015). "Categorías de una didáctica desarrolladora. Posición desde el enfoque histórico cultural». Educação e Filosofia, 29 (57): 61-93. DOI: 10.14393/REVEDFIL.issn.0102-6801. v29n57a2015-p61a93.

\section{Sobre el autor}

Mauricio Andrés Figueroa Mendoza es abogado. Magíster en Derecho por la Universidad Católica del Norte, Chile. Profesor de Historia y Geografía y Licenciado en Educación de la Universidad de Tarapacá, Chile. Diplomado en Educación Superior, Universidad Santo Tomás, Chile. Docente de la Escuela de Derecho en la Facultad de Derecho de Universidad Santo Tomás. Su correo electrónico es mfigueroa13@ santotomas.cl. (D) https://orcid.org/oooo-0oo2-1196-9904. 
La Revista Pedagogía Universitaria y Didáctica del Derecho (RPUDD) es una publicación científica semestral que contribuye a la reflexión multidisciplinaria sobre pedagogía universitaria y didáctica del derecho, para la formación y consolidación de esta área de investigación; así como a la difusión de prácticas innovadoras en la enseñanza-aprendizaje del derecho considerando el contexto nacional e internacional. Es una publicación electrónica internacional con una codirección entre Brasil y Chile.

\author{
DIRECTORA \\ María Francisca Elgueta Rosas \\ Universidad de Chile \\ DIRECTOR \\ Renato Duro Dias \\ Universidad Federal de Rio Grande, Brasil \\ SITIO WEB \\ pedagogiaderecho.uchile.cl \\ CORREO ELECTRÓNICO \\ rpedagogia@derecho.uchile.cl \\ LICENCIA DE ESTE ARTÍ́CULO
}

Creative Commons Atribución Compartir Igual 4.0 Internacional

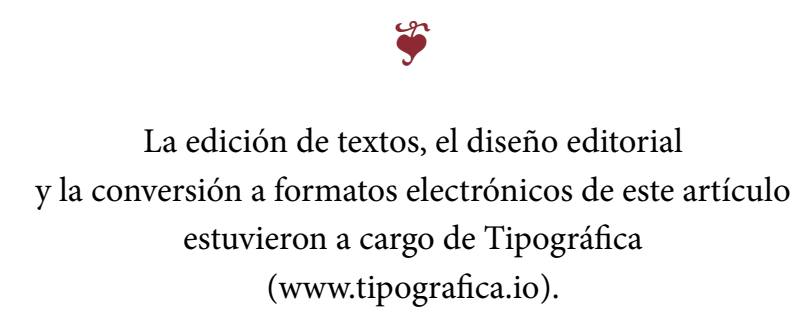

